Modeling, Identification and Control, Vol. 28, No. 3, 2007, pp. 81-86

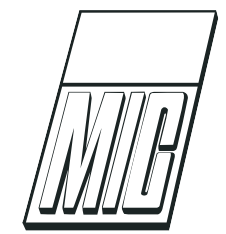

\title{
Improved Target Calculation for Model Predictive Control
}

\author{
Morten Hovd ${ }^{1}$ \\ ${ }^{1}$ Department of Engineering Cybernetics, Norwegian University of Science and Technology, N-7491 Trondheim, \\ Norway. E-mail: \{Morten.Hovd\} @itk.ntnu.no
}

\begin{abstract}
In industrial Model Predictive Control (MPC) applications, it is common to perform target calculation at each sample instant. The purpose of the target calculation is to translate operational targets supplied by higher level optimization functions into control targets that are feasible in the face of current disturbances. This paper shows that the commonly used target calculation formulation is flawed, and that this can lead to significant economic loss. A method for dealing with the identified problem is proposed*
\end{abstract}

Keywords: Model predictive control, target calculation, disturbances

\section{INTRODUCTION}

Model predictive control (MPC) is a hugely successful advanced control methodology, with thousands of implementations worldwide (Qin and Badgwell, 2003). $\mathrm{MPC}$ is also a very active research topic, at least since the well-known paper by Rawlings and Muske (1993). It is fair to say that MPC by now is well understood, at least for linear systems. However, a typical installation of MPC in a large-scale plant is only one of several layers in a control and decision hierarchy, such as the hierarchy illustrated in Fig. 1. In Fig. 1, MPC would fit in at the Supervisory Control layer. Although the presence of a control hierarchy is acknowledged in some control textbooks (e.g., Skogestad and Postlethwaite (1996)), it is typically ignored in most academic papers on MPC, in which the MPC control problem is typically addressed without consideration to how it is implemented in a control hierarchy such as Fig. 1.

The problem addressed in this paper arises primarily because of the timescale separation between different layers in Fig. 1. This timescale separation is a practical necessity when considering the objectives of the

\footnotetext{
*This paper is based on a paper presented at the IFAC Symposium DYCOPS, Cancun, Mexico, June 2007
}

different layers. The regulatory control layer operates at a timescale of seconds. It typically consists mainly of PI or PID controllers, and the required calculations are very simple, but are performed very frequently. The supervisory control (MPC) typically operates at a timescale of minutes, and solves an optimization problem (LP or QP) on line for each execution. Recent developments in Explicit MPC based on parametric programming (e.g., Bemporad et al. (2002)) does have the potential for reducing the on-line computational load and thereby enable increased sampling rates for MPC, but seem to have limited applicability to large-scale systems such as those typically found un the processing industries. The local optimization (often termed Real Time Optimization - RTO) typically operates at a timescale of hours. Whereas MPC (still) typically uses a linear dynamical model - some times with nonlinear transformations of inputs and/or outputs - RTO typically uses a static non-linear model derived from physical and chemical relationships.

The use of a static model for RTO means that after a disturbance has entered the system, the RTO has to wait for a new steady state to be reached before new control targets can be calculated. This 'steady state wait' has been identified by both industrialists 


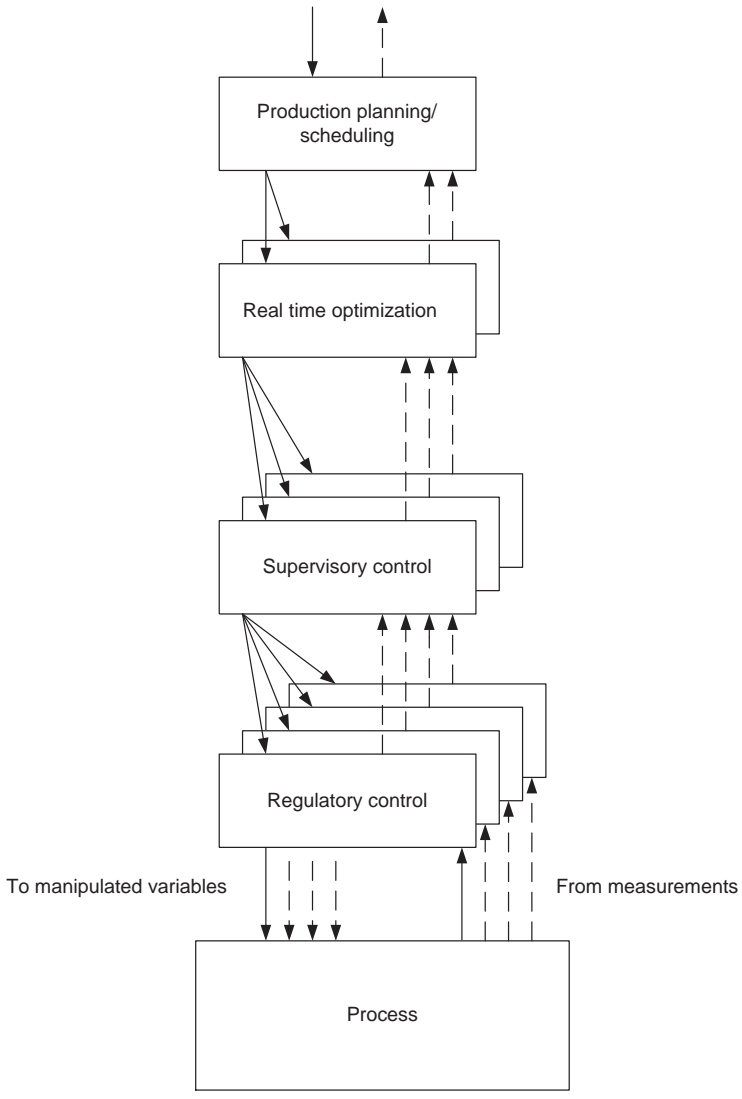

Figure 1: Control hierarchy in a large industrial plant.

(Friedman, 1995) and academics (Marlin and Hrymak, 1997) as one of the main drawbacks with RTO systems.

Industrial practitioners have not been able to afford the academic luxury of defining what problem to study. They have therefore had to address the fact that the MPC controller may be supplied with operational targets that are out of date or misleading. Therefore, industrial MPC applications commonly include a target calculation functionality. The objectives of the target calculation may be summarized thus:

- The operational targets may be specified in other variables than the control targets used by the MPC. The target calculation may therefore have to translate from operational targets to control targets.

- The number of variables specified may be different at different layers. The target calculation may therefore have to find an optimal tradeoff between the supplied operational targets (for over-determined systems), or to fix any unspecified degree of freedom (for under-determined systems).
- The operational targets supplied may specify an operating point that is not feasible, due to plantmodel mismatch or because disturbances have changed since the operational targets were last calculated. The target calculation then finds a feasible operating point as 'close' as possible to the specified operational targets.

Although target calculation has not received much academic attention, there are some notable exceptions. de Prada and Valentin (1996) provide an early contribution. Muske (1997) describes how the target calculation is formulated as a static QP problem, and discusses requirements for the existence of unique solutions of the QP problem. Rawlings (2000) briefly discusses target calculation in his tutorial on MPC. Ying and Joseph (1999) show how the weights used in the target calculation can be related to the plant economics as expressed by the RTO problem, and proves the stability of the overall system when the target calculation operates in cascade with a 'conventional' MPC. References to additional earlier works can be found in (Ying and Joseph, 1999).

The contribution of this paper is related to the latter of the target calculation objectives listed above. The main point is that changing disturbances generally will change the optimal operating point. Finding a feasible operating point as close as possible to the operational targets determined by the RTO (based on outdated disturbance data) can therefore lead to significant economic losses. The technique used for tracking the optimal steady state operating point is closely related to ideas presented in Kadam and Marquardt (2004) for RTO. Here, this is presented in the context of target calculation for MPC, and applied to an illustrative example.

\section{EXAMPLE}

In this section we will illustrate the problems of the conventional formulation of target calculation, using a reactor-separator-recycle process that has previously been studied by several authors, see Larsson et al. (2003). A diagram of the plant is shown in Fig. 2. In the reactor, component $A$ is converted to component $B$. Unreacted $A$ is separated in the separator (distillation column) and returned to the reactor. In this paper, a control structure described in (Larsson et al., 2003) as the 'Luyben rule' is used. This means that one of the flowrates in the recycle loop is fixed, in this case this is $F$, the feed rate to the distillation column. The other regulatory controls are:

- The reactor holdup $M_{r}$ is controlled using the distillate flowrate $D$. Larsson et al. (2003) found 


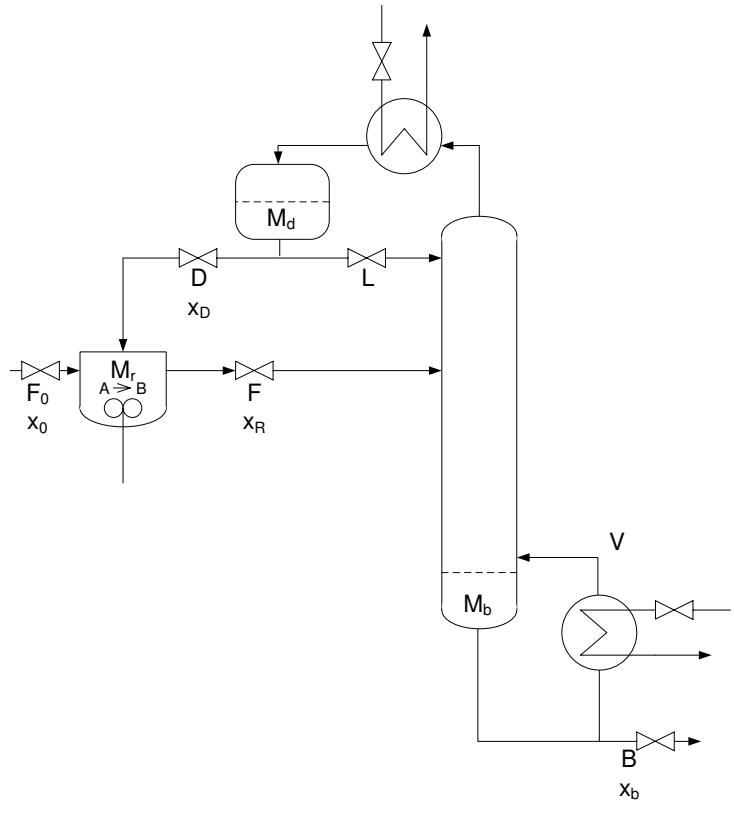

Figure 2: Diagram of reactor-separator-recycle plant.

that it was always optimal to keep the reactor holdup at its maximum $(2800 \mathrm{Kmol})$.

- The product composition $x_{B}$ is controlled using the boilup $V$. It is always optimal to have maximum allowable impurity in the product, which is a mole fraction of $A$ of 0.01015 .

- The condenser holdup $M_{D}$ is controlled using the reflux flowrate $L$.

- The column bottom holdup $M_{B}$ is controlled using the bottoms product flowrate $B$.

This control structure is not necessarily optimal for this plant, but the resulting closed loop system shows behavior which nicely illustrates the need for improved target calculation.

The external feed rate $F_{0}$ is here considered as a disturbance. When the external feed $F_{0}$ is determined elsewhere, optimal operation is achieved by minimizing the energy cost, i.e. by minimizing the boilup $V$ subject to product and operational constraints. In addition to the reactor holdup and product purity constraints, all flows are restricted to be non-negative, and there is a maximum boilup capacity such that $V \leq 5000 \mathrm{Kmol} / \mathrm{h}$. The nominal external feedrate is $F_{0}=460 \mathrm{Kmol} / \mathrm{h}$, with a possible disturbance range of $20 \%$. Figure 2 shows the relationships between feedrate $F$ to the column and the resulting boilup $V$ for the nominal and maximal $F_{0}$. Figure 2 nicely illus-

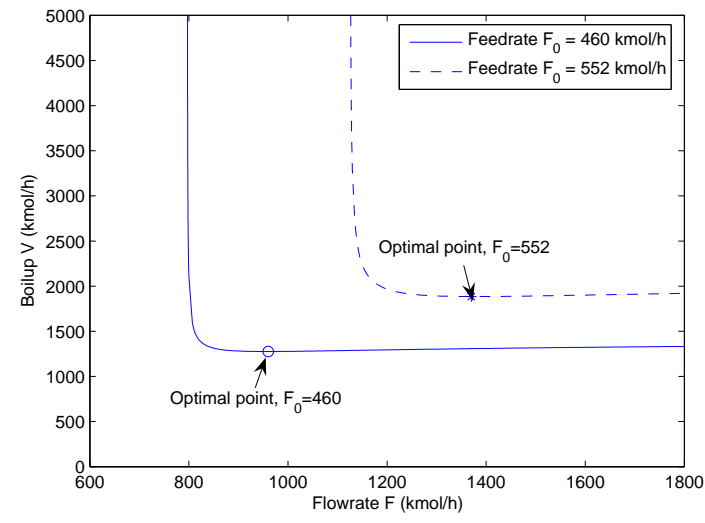

Figure 3: Boilup $V$ as a function of column feed $F$, for nominal and maximal external feed $F_{0}$.

trates the problem with trying to find a feasible operating point as close as possible to the nominally optimal operating point. For high values of $F_{0}$ this would result in a boilup of $V=5000 \mathrm{Kmol} / \mathrm{h}$, while the optimal boilup is well below $2000 \mathrm{Kmol} / \mathrm{h}$. This lead Govatsmark (2003) to propose the use of robust setpoints for this plant, where the robustness comes from optimizing the setpoint for the worst-case disturbance. Here that would lead to a constant column feedrate of $1370 \mathrm{Kmol} / \mathrm{h}$, and (for the maximal $F_{0}$ ) a corresponding boilup of $1885 \mathrm{Kmol} / \mathrm{h}$. Due to the shape of the objective function (the boilup curves in Fig. 2 are very flat to the right of the optimum) robust setpoints would work well in this case. For the nominal $F_{0}$ the robust setpoint for $F$ will give a boilup of $1308 \mathrm{Kmol} / h$, whereas the optimal value for $F$ gives a boilup of $1276 \mathrm{Kmol} / \mathrm{h}$.

However, one cannot in general expect to have such benign shapes of the objective function for RTO, and one must expect to incur severe losses by choosing a constant (albeit robustly optimal) control target. One would therefore like to be able to better adjust for changing disturbances in the target calculation, without having to wait for a new steady state which enables the RTO to execute. On the other hand, improvements to the target calculation should not significantly increase the computational complexity, in practice the target calculation should be no more complex than a $\mathrm{QP}$ of quite modest size (significantly smaller than the main QP in the MPC). 


\section{FORMULATION OF THE TARGET CALCULATION}

Following Ying and Joseph (1999), a typical target calculation problem may be formulated as:

$$
\begin{aligned}
\min _{z} & \frac{1}{2} z^{T} Q z+h^{T} z \\
\tilde{y} & =G u+w \\
\tilde{y}_{\min } \leq & \tilde{y} \leq \tilde{y}_{\max } \\
u_{\min } \leq & \leq \leq u_{\max }
\end{aligned}
$$

where $z^{T}=\left[\begin{array}{ll}y^{T} & u^{T}\end{array}\right]$ is the vector of control targets to be calculated, possibly consisting of both manipulated variables and plant outputs. The measurement vector $\tilde{y}$ consists both of those plant outputs $y$ whose optimal values are found in the target calculation, and any other plant outputs that may be constrained. In the example in the preceding section, only the column feedrate is determined by the target calculation, while there are also other plant flowrates that are constrained to be non-negative. Equation (2) ensures that the targets found are consistent with the steady-state plant model, and the vector $w$ accounts for the presently observed plant-model mismatch (whatever the cause may be). Both $u$ and $\tilde{y}$ are expressed in deviation variables, with the nominally optimal point as the origin.

Most elements in (1) - (4) would change with a changing nominal point (i.e., with a changing 'nominal' disturbance). The challenge will be to capture this changing problem description, while still retaining a relatively simple problem formulation. Here this problem is approached by attempting to model how the RTO problem changes with changing ("nominal") disturbances around the present nominal operating point,

To his end, the origin of the deviation variables is expressed as a series expansion in the observed disturbance $d$. Let $\tilde{y}^{*}$ and $u^{*}$ represent the optimal values of $\tilde{y}$ and $u$ for the nominal (for the RTO problem) disturbance value $d^{*}$. Then, the origin for the deviation variables $\tilde{y}$ and $u$ is for each vector element $i$ expressed as

$$
\begin{aligned}
& \tilde{y}_{i}^{0}=\tilde{y}_{i}^{*}+\frac{\partial \tilde{y}_{i}^{*}}{\partial d} \delta d+0.5 \delta d^{T} \frac{\partial \partial \tilde{y}_{i}^{*}}{\partial \partial d} \delta d+\cdots \\
& u_{i}^{0}=u_{i}^{*}+\frac{\partial u_{i}^{*}}{\partial d} \delta d+0.5 \delta d^{T} \frac{\partial \partial u_{i}^{*}}{\partial \partial d} \delta d+\cdots
\end{aligned}
$$

where the partial derivatives are evaluated at $d=d^{*}$, $u=u^{*}$. Note that making the origin of the deviation variables $\tilde{y}$ and $u$ dependent on $d$ means that the upper and lower limits in limits (3) and (4) will also change with $d$ (since they are fixed in terms of physical values). Next, it is necessary to express $Q, h$ and $G$ as a series expansion in terms of the observed disturbance $d$. This is done in a manner analogous to $(5,6)$.

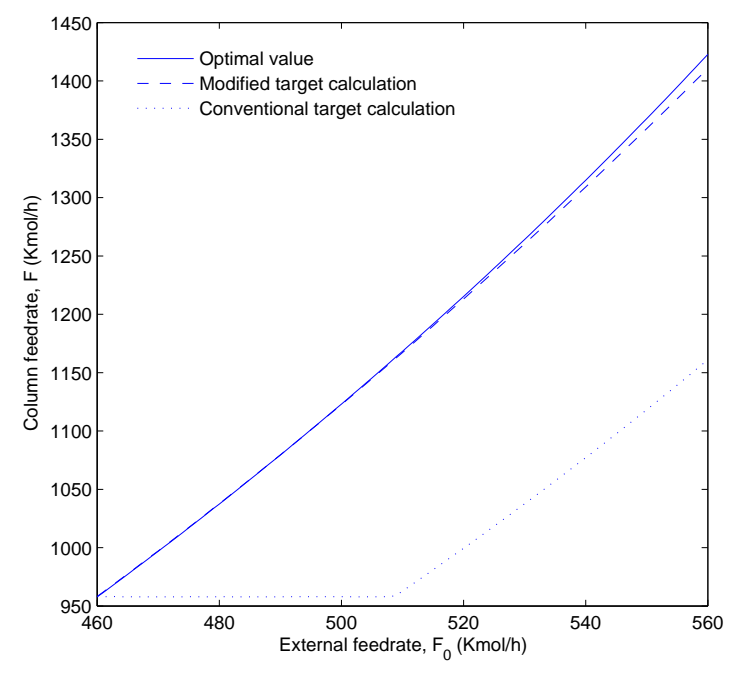

Figure 4: Column feedrate $F$, as function of external feedrate $F_{0}$.

\section{EXAMPLE REVISITED}

In section 2 we saw how and why the conventional formulation of the target calculation performs poorly for a large disturbance in the external flowrate $F_{0}$. Here the modified target calculation formulation of section 3 is investigated for the reactor-separator-recycle example, and compared to the conventional target calculation formulation.

From Fig. 4 it is clear that the modified target calculation formulation determines a column feedrate that is very close to that resulting from an optimization on the full non-linear model (denoted 'Optimal value' in Fig. 4). The conventional target calculation, on the other hand, tries to keep the column feedrate as close as possible to the optimal column feedrate for the nominal value of the disturbance (which in this case is $F_{0}=460 \mathrm{Kmol} / \mathrm{h}$ ). Only when the maximum boilup constraint is reached, will the conventional target calculation change the column feedrate target from the nominally optimal.

The resulting values for column boilup are shown in Fig. 5. On the scale of Fig. 5, the optimal boilup and the boilup resulting from the modified target calculation are virtually indistinguishable, whereas the conventional target calculation results in excessive boilup, i.e., a significant loss. 


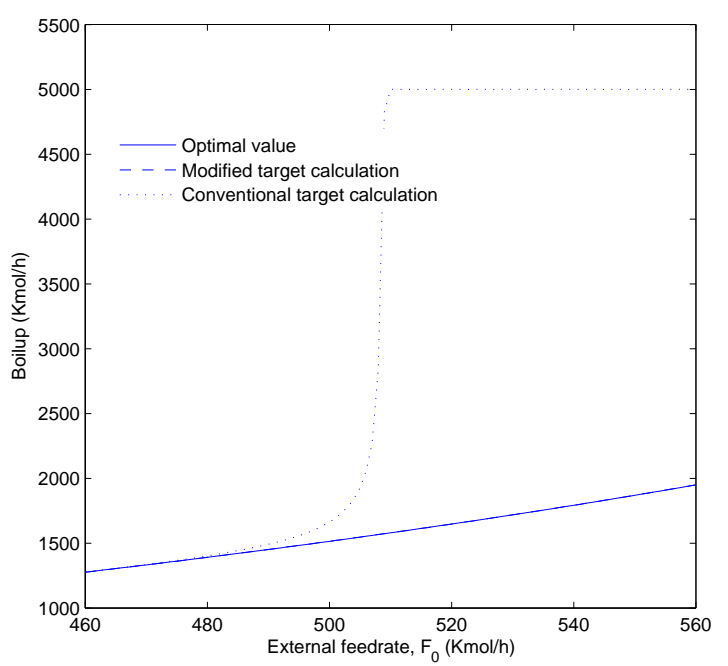

Figure 5: Column boilup $V$, as function of external feedrate $F_{0}$.

\section{DISCUSSION AND CONCLUSION}

This paper proposes a modification to the target calculation in MPC. Instead of finding a feasible operating point as close as possible to the nominally 'optimal' operating point in the face of changing disturbances, one should take into account that the location of the optimal operating point will typically also change when disturbances change. The modified target calculation attempts to account for both changes in the location of the optimal operating point, changes in the shape of the objective function, and changes in the constraints resulting from changing disturbances. The modified target calculation requires more information from the RTO layer than what is required for conventional target calculation. In addition to the nominally optimal control targets and the local shape of the objective function, information about how these change with changing disturbances is also required. This information may be obtained by perturbing the input data (disturbance values) for the RTO problem around the nominal value.

A critical issue for the application of the proposed modified target calculation is the ability to measure or reliably estimate the value of the disturbances (or at least the more important ones). Without reliable disturbance values there can be no hope of tracking the changes in the optimal operating point as the disturbances vary. In the example studied, the external feed flowrate is the main disturbance (and the only disturbance considered). Flowrates of gases and liquids are usually easily and reliably measured. However, feed composition disturbances are common in the processing industries - and composition measurements can be costly, cumbersome and unreliable. Whether secondary measurements can be used to estimate hardto-measure disturbances is of course totally problem dependent.

It is also important to have a sober understanding of what disturbances should result in changes in the control targets. Clearly, it does not make sense to change the control targets due to high-frequency disturbances. Only disturbance components that are well within the bandwidth of the MPC should result in changed control targets for the MPC. This implies filtering of disturbances prior to target calculation. Such filtering may be built into the estimator for disturbances that are not measured directly.

\section{ACKNOWLEDGEMENT}

The author wishes to thank Dr. M. S. Govatsmark of Cybernetica AS for access to his model of the reactorseparator-recycle process.

\section{References}

Bemporad, A., Morari, M., Dua, V., and Pistikopoulos, E. N. The explicit linear quadratic regulator for constrained systems. Automatica, 2002. 38:3-20.

Friedman, Y. What's wrong with unit closed loop optimization. Hydrocarbon Processing, 1995. (October):107 - 116 .

Govatsmark, M. Integrated Optimization and Control. Ph.D. thesis, Norwegian University of Science and Technology, Dept. of Chemical Engineering, 2003.

Kadam, J. and Marquardt, W. Sensitivity-based solution updates in closed-loop dynamic optimization. In Proceedings of the DYCOPS 7 conference. 2004 .

Larsson, T., Govatsmark, M., Skogestad, S., and Yu, C. Control structure selection for reactor, separator and recycle process. Ind. Eng. Chem. Res., 2003. 42.

Marlin, T. and Hrymak, A. N. Real-time operations optimization of continuous processes. AIChE Symposium Series, 1997. (93):156 - 164.

Muske, K. R. Steady-state target optimization in linear model predictive control. In Proc. American Control Conference. 1997 pages 3597-3601. 
de Prada, C. and Valentin, A. Set point optimization in multivariable constrained predictive control. In Proc. 13th IFAC World Congress. San Francisco, California, 1996 .

Qin, S. J. and Badgwell, T. A. A survey of industrial model predictive control technology. Control Engineering Practice, 2003. pages 733-764.

Rawlings, J. B. Tutorial overview of model predictive control. IEEE Control Systems Magazine, 2000. $20(3): 38-52$.

Rawlings, J. B. and Muske, K. R. The stability of constrained receding horizon control. IEEE Transactions on Automatic Control, 1993. 38(10):15121516.

Skogestad, S. and Postlethwaite, I. Multivariable Feedback Control. Analysis and Design. John Wiley \& Sons Ltd, Chichester, England, 1996.

Ying, C.-M. and Joseph, B. Performance and stability analysis of lp-mpc and qp-mpc cascade control systems. AIChE Journal, 1999. 45(7):1521-1534. 